\title{
How Safe is the Use of Intrathecal Fluorescein? An Experimental Study
}

\author{
Mahmut CAMLAR ${ }^{1}$, Caglar TURK¹, Fatih OLTULU², Merve OREN ${ }^{3}$, Aylin BUHUR², Gurkan YIGITTURK², \\ Fusun OZER ${ }^{1}$, Nurperi GAZIOGLU ${ }^{4}$ \\ 'University of Health Sciences, Izmir Tepecik Training and Research Hospital, Department of Neurosurgery, Izmir, Turkey \\ ${ }^{2}$ Ege University, Histology and Embryology Department, Bornova, Izmir, Turkey \\ ${ }^{3}$ Erzurum Provincial Health Directorate, Public Health Services Presidency, Erzurum, Turkey \\ ${ }^{4}$ Istanbul Bilim University, Department of Neurosurgery, Istanbul, Turkey
}

Corresponding author: Mahmut CAMLAR drcamlar@gmail.com

\section{ABSTRACT}

AIM: To investigate the effects of fluorescein-sodium on neural tissues.

MATERIAL and METHODS: Twenty-one Wistar rats were randomly divided into three experimental groups: control (group 1) and fluorescein-sodium groups with different doses (groups 2 and 3). In the control group, craniectomy following with durotomy was performed with the help of a loupe microscope, and a dry sponge was overlayed to the brain tissue. In the study groups, the open dura was covered with a sponge soaked with $0.02 \mathrm{mg}$ (group 2) and with $0.2 \mathrm{mg}$ (group 3) fluorescein sodium following craniectomy. Three weeks postoperatively, rats were sacrificed for the histopathologic evaluations.

RESULTS: Fluorescein-induced apoptosis occurs in a dose-dependent manner in rats' neurons. It was determined that neuron and neuroglial cell TUNEL staining was statistically different among the three groups $(p<0.001)$. Our results indicated that fluorescein induces apoptosis, resulting in increased nuclear factor kappa beta (NF-k $\beta$ ) expression in a dose-dependent manner.

CONCLUSION: Fluorescein sodium is used frequently during surgery for CSF fistulas. However, information in the literature about its safety is insufficient. Our study holds promise for the development of new studies on the reliability of this agent.

KEYWORDS: Fluorescein-sodium, Rhinorrhea, Neurotoxic effect, CSF fistula

ABBREVIATIONS: CSF: Cerebrospinal fluid, NF-к $\beta$ : Nuclear factor kappa beta, IP: Intraperitoneal, H-score: Histoscore, PBS: Phosphate-buffered saline, TdT: Terminal deoxy-nucleotidyl transferase, H\&E: Hematoxylin and eosin, Al: Apoptotic index,

DAB: 3',3'-diaminobenzidine

\section{INTRODUCTION}

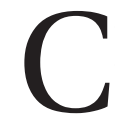
erebrospinal fluid (CSF) leaks can be caused by many different etiologies, most commonly history of trauma, iatrogenic or nontraumatic bone invasions, empty sella syndrome, congenital bone defects, infections and meningoencephalocele, meningeal diverticula, and dural sac weakness. Diagnosis of CSF rhinorrhea starts with the wellinterviewed patient history and continues with the confirmation of the onset of leakage with the help of laboratory tests. $\beta 2$ Transferrin which is also known as Tau protein is an isoform of transferrin and only found in perilymphatic fluid and CSF. With the help of this parameter, some patients who do not have genuine CSF leakage do not undergo unnecessary invasive tests (13). After the detection of $\beta 2$-transferrin, patients must undergo endoscopic examination to determine the dural defect. The source of leakage may be from the different parts
Mahmut CAMLAR (1D : 0000-0002-6467-0172

Caglar TURK (D : 0000-0002-2768-2861

Fatih OLTULU (1D : 0000-0001-6475-642X $\begin{array}{ll}\text { Merve OREN } & \text { (D) : 0000-0002-3383-7830 } \\ \text { Aylin BUHUR } & \text { (D): 0000-0002-3759-6616 }\end{array}$

Gurkan YIGITTURK (D) : 0000-0002-5315-253X
Fusun OZER

Nurperi GAZIOGLU

(1) : $0000-0002-1625-5302$

(D) : $0000-0001-7785-8628$ 
of the cranial fossa; anterior, middle or posterior. Traumatic or incidental fractures are found mostly at the cribriform plate which has adherent duramater $(9,10)$.

Many treatment methods have been defined for management of CSF by neurosurgeons and otorhinolaryngologists. Bed rest, laxative treatment, and lumbar drainage are some defined treatment methods. Some studies report that these leaks carry a meningitis risk as high as $30 \%$ and should be repaired $(3,4)$. Although transsphenoidal and transcranial approaches have been defined for repair of dural defects, an endoscopic transnasal transsphenoidal approach is mostly preferred in the anterior skull base. This approach is less invasive and has achieved successful results with low complication rates (12).

Fluorescein sodium is commonly used by ophthalmologists to detect intractable structures of the human eye. At some centers, the same dye is frequently used by neurosurgeons and otorhinolaryngologists to detect CSF fistulas with very successful results (17). We investigated the changes in brain tissue with use of fluorescein and determined its relationship with these complications. Although some complications have been reported, an experimental study showing the histologic effects of fluorescein has not been performed previously to our knowledge. We believe our work will shed light on this issue.

\section{MATERIAL and METHODS}

Ege University Animal Research Ethics Committee approval was obtained for our experimental study and performed according to the "Guide for the Care and Use of Laboratory Animals" in the Animal Laboratory (ARGEFAR) of Ege University (approval number/date 2017-001/22.02.2017). We used the similar model from our prior study on the neurotoxic effects of povidone-iodine (2). In daily life during the operation, fluorescein was administered intrathecally from the lumbar region of the patient with catheters placed intrathecally in Trendelenburg position, allowing fluorescein to flow into the intracranial compartment. Since the subarachnoid space in mice was quite narrow, the fluorescein could not be confirmed to reach the intracranial compartment and we believed it more appropriate to apply it directly to the intracranial compartment, contacting brain tissue directly in the intracranial subarachnoid area.

Twenty-one adult Wistar albino rats weighing $350 \mathrm{~g}$ were housed in separate cages with a standard laboratory conditions. Three groups of 7 rats ( 4 male, 3 female) were randomly selected. Group 1 was the control group, while rats in groups 2 and 3 were given fluorescein sodium at different doses.

On day 21, rats were anesthetized with an intraperitoneal (IP) ketamine hydrochloride-xylazine combination (5-10 mg/ $\mathrm{kg}$ ) and $15 \mathrm{~g} / \mathrm{kg}$ subcutaneous cefazolin sodium was given prophylactically 1 hour preoperatively. After positioning and fixation, surgery was performed under sterile conditions using a loupe microscope. A linear scalp incision was performed on the right frontal midpupillary line. A burr hole and nearly $1 \times 1 \mathrm{~cm}$ craniectomy were performed with a lancet and micro-surgical set. A dural incision was made using microtweezers and a microscalpel. In Group 1, the dural defect was covered with a Spongostan (Ethicon, Inc., Somerville, NJ, USA), a hemostatic agent used in neurosurgical operations, and then the skin was sutured with $3 / 0$ polypropylene. The dural defect was covered with a Spongostan soaked with $0.02 \mathrm{mg}$ (approximately 4.5 $\mathrm{mg} / 75 \mathrm{~kg}$; group 2) and $0.2 \mathrm{mg}$ (approximately $45 \mathrm{mg} / 75 \mathrm{~kg}$; group 3) fluorescein. Skin was sutured in the same manner. Rats were placed back in their cages separately and housed for 3 more weeks.

\section{Histologic Examination}

IP $40 \mathrm{mg} / \mathrm{kg}$ ketamine and $4 \mathrm{mg} / \mathrm{kg}$ xylazine injection is performed for the anesthesia of the animals and fixed with $4 \%$ formaldehyde in $0.1 \mathrm{M}$ phosphate-buffered saline (PBS) perfusion. Hematoxylin and eosin (H\&E) staining system was performed to stain the $5 \mathrm{~mm}$ sectioned cerebrum paraffin blocks. Photographs of sections were obtained with Olympus C-5050 digital camera (Olympus Optical Co.,Tokyo, Japan).

\section{Immunohistochemistry}

We used the similar immunohistochemistry protocol of the studies performed by Acikgoz et al. and Oktem et al. for our study $(1,14)$. Motorized rotary microtome (MR-2145; Leica Biosystems, Wetzlar, Germany) was used to cut the $5 \mathrm{~mm}$ thick sections. Sections were washed with phosphate-buffered saline and treated with 2\% trypsin (Sigma-Aldrich Corp., St. Louis, USA) for 15 minutes in $50 \mu \mathrm{M}$ Tris-buffer following delineation with a Dako-pen (Dako, Glostrup, Denmark). Hydrogen peroxide $\left(\mathrm{H}_{2} \mathrm{O}_{2}\right)$ (3\%) was used to inhibit the peroxidase activity. Next step was the incubation of sections with the primary antibodies against nuclear factor kappa beta (NF-к $\beta ; 1 / 100)$ for 24 hours and then with bio-tinylated secondary antibody following streptavid in conjugated to horseradish peroxidase (Zymed Histostain-Plus peroxidase kit; 85-9043; Zymed Laboratories, San Francisco, CA, USA). For the detection of apoptosis sections were incubated with DAB for 5 minutes to observe immune labeling (DAB; DeadEnd Colorimetric [TUNEL] system; Promega, Madison, WI, USA). Sections were counterstained with Mayer's hematoxylin (Zymed Laboratories) and were dehydrated with a graded ethanol cleared in xylene, and mounted with the help of Entellan (Merck, Darmstadt, Germany). Three histologists independently assessed the positive cells. Scores were calculated as; 100 cells per 10 view-fields of sections at $\times 20$ magnification and the photographs were obtained using Olympus C-5050 camera.

$\mathrm{NF}-\kappa \beta$ immunohistochemical semiquantitation was performed using the modified Histoscore which was calculated by the positive cells percentage and a semi-quantitative assessment of the intensity of staining (non-staining: 0 , weak: 1 , median: 2, strong: 3). The scores may range from 0 to 300 . The expression rates were determined as low and high, depending to the Histoscore results.

\section{TUNEL Assay}

Deparaffinized sections were incubated with proteinase $\mathrm{K}(20$ $\mu \mathrm{MmL}^{-1}$ ) for 10 minutes. $\mathrm{H}_{2} \mathrm{O}_{2}(3 \%)$ was used for the inhibition 
for the peroxidase activity. Next step was the incubation with equilibration buffer, Terminal deoxynucleotidyl transferase (TdT) enzyme (1 hour) and finally with anistreptavidinperoxidase for 45-50 minutes. Staining with $D A B$ and counterstaining with Mayers hematoxylin was performed. Quantification of apoptotic cells was performed by at least two independent observers and apoptotic cells were counted with $\times 40$ magnification of a light microscope. Apoptotic index (Al) was defined as apoptotic cell count per section (Al = total apoptotic cell count/100).

\section{Statistical Analysis}

Descriptive statistics are reported as median with interquartile range (IQR) values. The variables were investigated using analytical methods (Schapiro-Wilk's test) to determine whether they were normally distributed. Kruskal-Wallis tests were used for statistical comparison of data between groups. To check the significance of pairwise differences The MannWhitney $U$ test was performed using correction of Bonferroni to adjust for multiple comparisons. Spearman's correlation and Kendall's Tau-b correlation were run to determine the relationship between Groups 1, 2, and 3, and TUNEL/NF-к $\beta$ positive staining cell number values. In the interpretation of the correlation coefficient, $0.0-0.24$ weak, $0.25-0.49$ medium, 0.50-0.74 strong, $0.75-1.00$ very strong. For statistical significance, $\mathrm{p}<0.05$ was considered as significant in the 95\% confidence interval. Statistical analyses were performed using Statistical Package for the Social Sciences (SPSS, Inc., Chicago, IL, USA) program, version 21.0.

\section{RESULTS}

The median (IQR) numbers of neuroglial cells detected by $N F-\kappa \beta$ staining was 45 (6), 28 (2), and 30 (6) in groups 1-3, respectively. The number of neurons stained with NF- $\mathrm{k} \beta$ was 8 (2), 5 (2), and 3 (1), respectively (Figure 1A-C). The number of neuroglial cells stained with TUNEL were 19 (4), 9 (3), and 2 (1), respectively, while the number of neurons stained with TUNEL were 5 (2), $2(0)$, and $1(1)$, respectively (Figure 2A-C). Neuron and neuroglial cell staining was statistically different among the three groups in the type of TUNEL staining $(p<0.001$; Figure 3).

Post hoc analyses of these results showed that the number of neuroglial cells stained with NF- $\kappa \beta$ was higher in Group 1 than in the other groups $(p=0.001, p=0.019$ for groups 2 and 3 , respectively). The number of neurons stained with NF- $\kappa \beta$ and the number of neurons and neuroglial cells stained with TUNEL were significantly lower in the control groups (both statistically significant, $p<0.001$; Table I).

Correlation analysis was performed to demonstrate increased toxicity with increasing doses of fluorescein. A very strong and significant correlation was found between the dose increase in the groups and the neuron and neuroglia TUNEL staining in the positive direction ( $r=0.838,0.855 ; p<0.001$, respectively). Apoptosis rate increased as fluorescein dose increased.

Increased doses of fluorescein showed a moderate strong correlation between $\mathrm{NF}-\kappa \beta-$ positive stained neuroglial cells
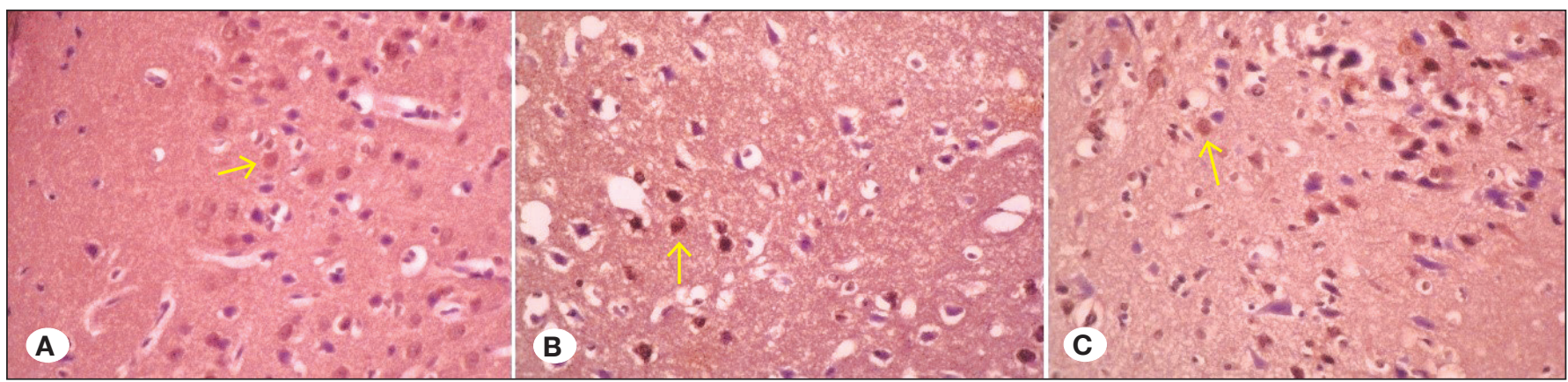

Figure 1: Immunohistochemical image of the change in NF-к $\beta$ protein. Positive-stained cell nuclei appear to be stained dark brown. Yellow arrow marks one positive stained cell. Original magnification ×40 (A: Group 1, B: Group 2, C: Group 3).

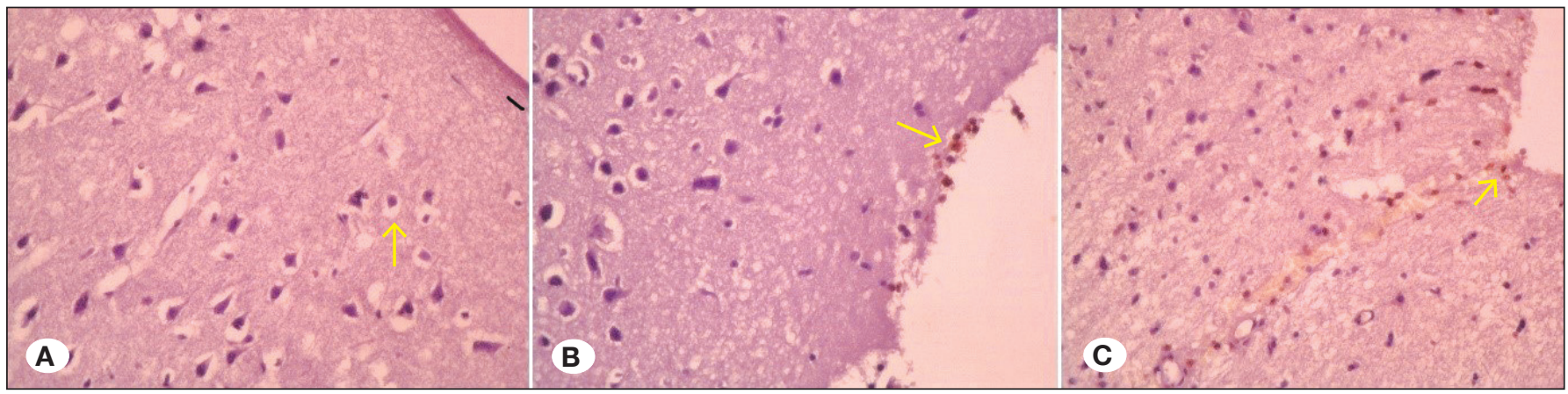

Figure 2: TUNEL assay results. Apoptotic cells appear to have brown-colored cytoplasm (yellow arrow). Original magnification $\times 40$ (A: Group 1, B: Group 2, C: Group 3). 
Table I: Statistical Results of the Groups: Median (IQR)

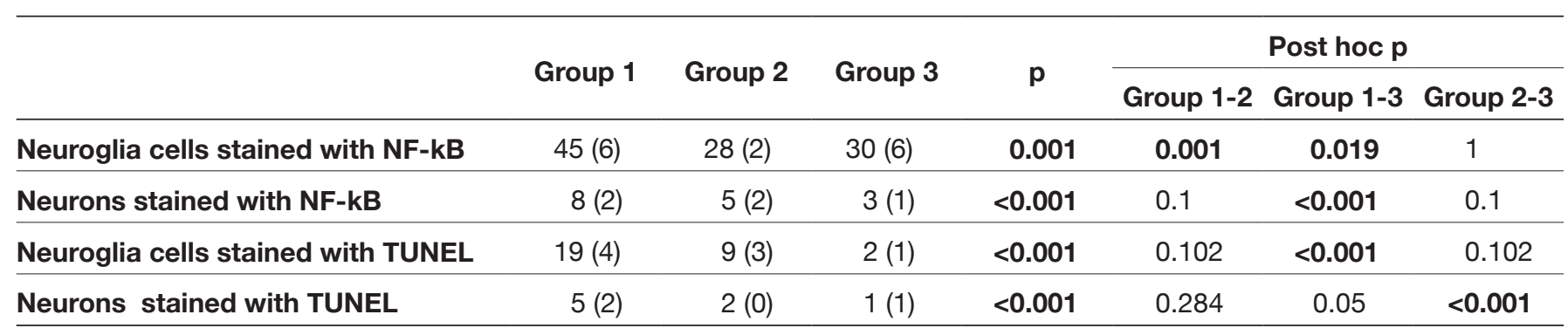
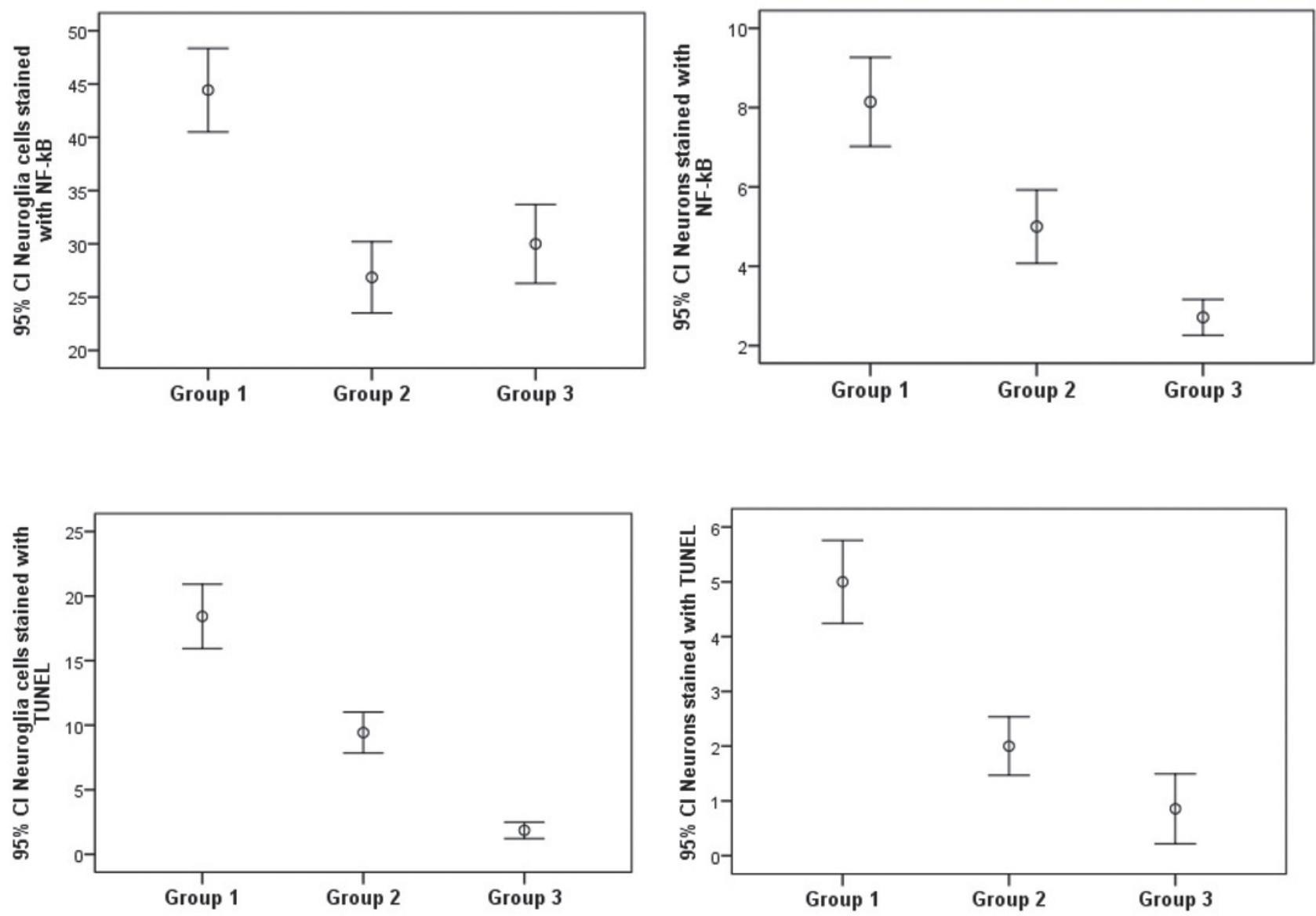

Figure 3: Statistical analysis results of neuron and neuroglial cells staining with NF-k $\beta$ and TUNEL.

and very strong correlation between neuronal cell numbers $(r=0.451, p=0.013$ and $r=0.882, p<0.001$, respectively).

\section{H\&E Staining}

In all groups, pyramidal cells, granular cells, and neuron bodies of fusiform cells, as well as central neuroglial cells, axonal extensions, and blood capillaries were distinguished in the gray matter region of the brain tissue. In the inner white matter region, axons extending from neurons in gray matter, glial cells associated with axons, and blood capillaries are observed. In Group 2, the relative increase in the number of neuroglial cells, especially in the gray matter region (groups 1 and 3 ), was noted (Figure 4A-C).

\section{DISCUSSION}

In 1960, intrathecal fluorescein was described by Kirchner and Proud to localize the origin of otorrhea and rhinorrhea (6). This technique is used if other imaging methods were insufficient for diagnosis or to observe the origin of leakage during surgery. In 1926, Dandy described the first case of CSF fistula repair (16). After this date, repair with craniotomy become widespread. 


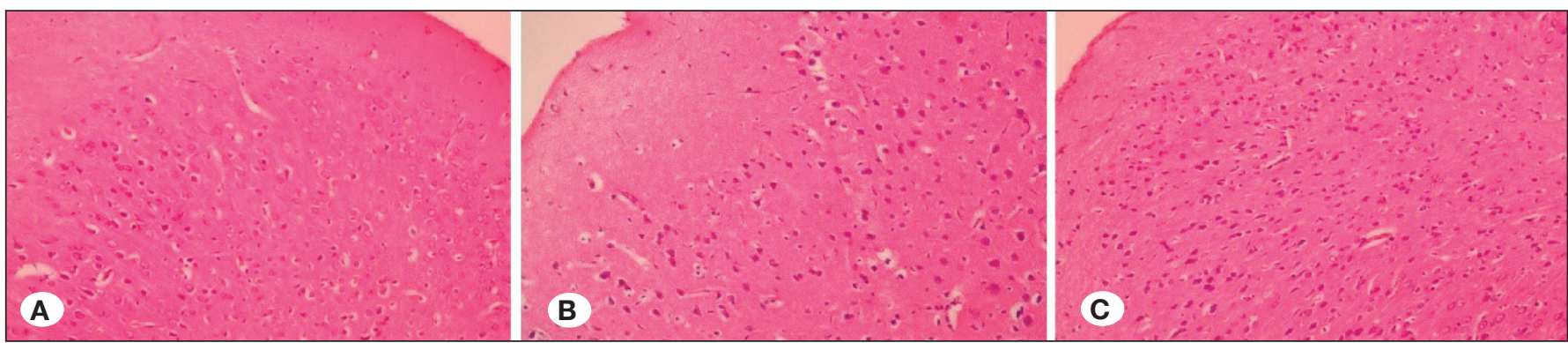

Figure 4: Histologic changes after fluorescein administration. Relative increase in the number of neuroglial cells observed. H\&E, original magnification ×20 (A: Group 1, B: Group 2, C: Group 3).

With time, endoscopic approaches were identified and became the gold standard, with a success rate of $60 \%$ to $100 \%$. The success of the process largely depends on the detection of the leakage source and the use of fluorescein has become widespread. However, there is no clear consensus in the literature about the reliability, dosage, and infusion details of this dye. Although various imaging modalities are ambitious in determining the leak location, some surgeons prefer to see the leakage during surgery.

Several side effects of using intrathecal fluorescein sodium have been reported to date. Color changes in tears and urine, headache and neck pain, nausea, vomiting, fever and dizziness, cranial nerve deficits, lower extremity weakness, and numbness are the most common side effects. In the literature, there are rare but more serious adverse effects and complications, such as epileptic seizures, opisthotonos, hemiparesis, dysphasia, myelopathy, pulmonary edema, and death $(3,6,9,11)$. The etiology and time of seizure activity remains unclear, suggesting that fluorescein may trigger direct central neuro-activation or meningeal irritation, or may have caused aseptic meningitis. Also, contamination of CSF with erythrocytes during lumbar drainage can cause seizure-like activities (6). In 1997, Wolf et al. described a large cohort of 925 patients (18) and reported fluorescein-related seizure in three patients (18). Although complications are rare, they can be serious. Some complications mentioned above may be due to lumbar puncture, but there are not enough data in this regard. In 1978, Moseley et al. surveyed the American Association of Neurological Surgeon members for the use of intrathecal fluorescein sodium and showed that all complications were transient (3). To avoid complications, John et al. described perioperative intranasal application of fluorescein sodium to the nasal cavity after exposure, reporting that yellow-colored fluorescein turns to green when it diffuses to CSF, so that the fistula is more easily observed (7).

Keerl et al. analyzed the results of 420 intrathecal application of fluorescein sodium solutions and the complications: in Germany, a maximum dose of $50 \mathrm{mg} / \mathrm{mL}$ fluorescein was used, while in the United States, a more dilated solution of $0.5-2 \mathrm{~mL}$ (2.5-10 mg) was used perioperatively (10). They observed two grand mal seizures in Germany, but no apparent side effects with this hypodense fluorescein solution in the United States. In our study, we set the fluorescein doses according to doses defined in their study. We also found that apoptosis increases depending on the increased dose of fluorescein, and that it also must be diluted before application due to high $\mathrm{pH}$.

$\mathrm{NF}-\kappa \beta$ is a well known protein structured transcription factor which has an important role in regulating cell activities, such as the expression of genes that promote cell viability, inflammation, apoptosis and cell proliferation (8). The active form of NF- $\kappa \beta$ acts like a transcription factor for target genes and can be induced by agents that damage DNA (15). In mammals, NF- $\kappa \beta$ has a structurally related proteins. Rel (c-Rel), RelA (p65), RelB, NF- $\kappa \beta 1$, and NF- $\kappa \beta 2$ are the members of this protein (5). Still there is no consensus about which of the target genes activated by this proteins are critical for the suppression process of apoptosis, while the inhibition of apoptosis by NF$\kappa \beta$ may vary from one cell type to another (7). Studies have shown that inflammatory process is mediated through NF$\mathrm{kB}$ activation and the agents with anti-inflammatory effect suppress the activation of NF- $\kappa \beta$. Paradoxically, toxic agents, cytokines, and radiation, which induce the apoptosis, also

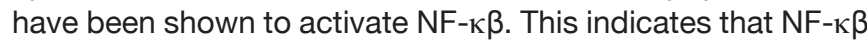
may be an important part of the cells' auto-defense system and may mediate chemo-radioresistance. The causes that activate the $N F-\kappa \beta$ found in tumor cells are misunderstood. In rare cases, NF- $\kappa \beta$ has a pro-apoptotic role despite its more common anti-apoptotic role. This opposing effect of NF-к $\beta$ might be related to cell type-specific and/or inducing signalto-cell factors. As a result, $N F-\kappa \beta$ activation with different pathways can trigger or inhibit apoptosis $(5,15)$. We observed that administration of fluorescein increased the expression of $N F-\kappa \beta$, while the increased doses of fluorescein showed a

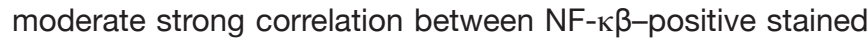
neuroglial cells and very strong correlation between neuronal cell numbers.

Intrathecal administration of fluorescein sodium may be an important diagnostic method in the localization of CSF leak. Before the procedure, the patient and anesthesiologist should be informed about the side effects and benefits of fluorescein to the surgical team, because intrathecal use of fluorescein is considered as "off-label" use (6).

In our study, comparing histologic changes and apoptosis between the fluorescein groups and the control group, apoptosis occurrence was statistically higher in the fluorescein groups. However, we found no statistically significant difference among the three groups in terms of pyramidal cells, 
granular cells, and neuron bodies of fusiform cells, as well as central neuroglial cells in the gray matter of the brain tissue and in the white matter region axons and glial cells. Attention should be given to the use of fluorescein when considering the effects on the cellular level and apoptosis. To our knowledge, this is the first experimental study regarding the toxic effect of fluorescein in the neural tissue.

\section{CONCLUSION}

Intrathecal fluorescein currently is being used at many centers and there is an emphasis that the safe dose should not be exceeded. Side effects, such as seizures, are known to occur at high doses, but to our knowledge, no animal experiments exist investigating these dose-related neurotoxicity complications. Our study showed that neuron and neuroglial cell staining was statistically different among the three groups in the type of tunneling $(p<0.001$; Figure 4$)$. Moreover, our results indicated that fluorescein induces apoptosis, resulting in increased NF$\kappa \beta$ expression in a dose-dependent manner.

\section{ACKNOWLEDGMENTS}

We thank Prof. Huseyin Aktug and Prof. Altug Yavasoglu for their significant contributions and special aids at histology and embryology department laboratories of Ege University.

\section{- REFERENCES}

1. Acikgoz E, Aktug H, Yigitturk G, Demir K, Guven U, Duzagac F, Oltulu F, Yavasoglu A, Oktem G: Repression of the Notch pathway prevents liver damage in streptozotocin-induced diabetic mice. Folia Histochem Cytobiol 55:140-148, 2017

2. Akcay E, Ersahin Y, Ozer F, Duransoy YK, Camlar M, Atci I, Yagci A, Ozer O: Neurotoxic effect of povidone-iodine on the rat spine using a laminectomy-durotomy model. Childs Nerv Syst 28:2071-2075, 2012

3. Anari S, Waldron M, Carrie S: Delayed absence seizure: A complication of intrathecal fluorescein injection. A case report and literature review. Auris Nasus Larynx 34:515-518, 2007

4. Fishman AJ, Hoffman RA, Roland JT Jr, Lebowitz RA, Cohen $\mathrm{NL}$ : CSF drainage in the management of CSF leak following acoustic neuroma surgery. Laryngoscope 106:1002-1004, 1996

5. Ghosh S, Karin M: Missing pieces in the NF-kappa B puzzle. Cell 109 Suppl:81-96, 2002
6. Jacob AK, Dilger JA, Hebl JR: Status epilepticus and intrathecal fluorescein: Anesthesia providers beware. Anesth Analg 107:229-231, 2008

7. Jones ME, Reino T, Gnoy A, Guillory S, Wackym Pi, Lawson W: Identification of intranasal cerebrospinal fluid leaks by topical application with fluorescein dye. Am J Rhinol 14:93-96, 2000

8. Karin $M$, Lin $A: N F-\kappa \beta$ at the crossroads of life and death. Nat Immunol 3:221-227, 2002

9. Keerl R, Weber RK, Draf W, Radziwill R, Wienke A: Complications of lumbar administration of $5 \%$ sodium fluorescein solution for detection of cerebrospinal fluid fistula. Laryngorhinootologie 82:833-838, 2003

10. Keerl R, Weber RK, Draf W, Wienke A, Schaefer SD: Use of sodium fluorescein solution for detection of cerebrospinal fluid fistulas: An analysis of 420 administrations and reported complications in Europe and the United States. Laryngoscope 114:266-272, 2004

11. Kerr JT, Chu FW, Bayles SW: Cerebrospinal fluid rhinorrhoea: Diagnosis and management. Otolaryngol Clin North Am 38:597-611, 2005

12. Marshall AH, Jones NS, Robertson IJ: CSF rhinorrhoea: The place of endoscopic surgery. Br J Neurosurg 15:8-12, 2001

13. Nandapalan V, Watson ID, Swift AC: Beta 2 transferrin and cerebrospinal fluid rhinorrhoea. Clin Otolaryngol Allied Sci 21:259-264, 1996

14. Oktem G, Uysal A, Oral O, Sezer ED, Olukman M, Erol A, Akgur SA, Bilir A: Resveratrol attenuates doxorubicin-induced cellular damage by modulating nitric oxide and apoptosis. Experimental and Toxicologic Pathology 64:471-479, 2010

15. Puliyappadamba VT, Hatanpaa KJ, Chakraborty S, Chakraborty S, Habib AA: The role of NF-kB in the pathogenesis of glioma. Mol Cell Oncol 1: e963478, 2014

16. Rodríguez-Navarro MÁ, Díaz-Alejo C, Padilla-Del Rey ML, Alcaraz AB, González-Pérez Pi, Benítez M: Safe intrathecal fluorescein use for identification of cerebrospinal fluid leaks: Case-report and perioperative algorithm description. Rev Esp Anestesiol Reanim 64:533-536, 2017

17. Schlosser R, Bolger WE: Endoscopic management of cerebrospinal fluid rhinorrhea. Otolaryngol Clin N Am 39:523538, 2006

18. Wolf G, Greistorfer K, Stammberger H: Endoscopic detection of cerebrospinal fluid fistulas with a fluorescence technique. Report of experiences with over 925 cases. Laryngorhinootologie 76:588-594, 1997 